\title{
Stability Analysis of Impulsive Stochastic Functional Differential Equations with Delayed Impulses via Comparison Principle and Impulsive Delay Differential Inequality
}

\author{
Pei Cheng, ${ }^{1}$ Fengqi Yao, ${ }^{2}$ and Mingang $\mathrm{Hua}^{3}$ \\ ${ }^{1}$ School of Mathematical Sciences, Anhui University, Hefei 230601, China \\ ${ }^{2}$ School of Electrical Engineering and Information, Anhui University of Technology, Ma'anshan 243000, China \\ ${ }^{3}$ College of Computer and Information, Hohai University, Changzhou 213022, China \\ Correspondence should be addressed to Pei Cheng; chengpei_pi@163.com
}

Received 25 July 2013; Revised 26 November 2013; Accepted 10 December 2013; Published 16 February 2014

Academic Editor: Yuriy Rogovchenko

Copyright (C) 2014 Pei Cheng et al. This is an open access article distributed under the Creative Commons Attribution License, which permits unrestricted use, distribution, and reproduction in any medium, provided the original work is properly cited.

\begin{abstract}
The problem of stability for nonlinear impulsive stochastic functional differential equations with delayed impulses is addressed in this paper. Based on the comparison principle and an impulsive delay differential inequality, some exponential stability and asymptotical stability criteria are derived, which show that the system will be stable if the impulses' frequency and amplitude are suitably related to the increase or decrease of the continuous stochastic flows. The obtained results complement ones from some recent works. Two examples are discussed to illustrate the effectiveness and advantages of our results.
\end{abstract}

\section{Introduction}

Impulsive dynamical equations have received considerable attention during the recent decades since they provide a natural framework for mathematical modeling of many real world evolutionary processes where the states undergo abrupt changes at certain instants (see [1-7]). In particular, more researchers have given special interests to the stability and stabilization analysis of impulsive functional differential equations (IFDEs) and there are extensive literatures in this field (see [8-14] and reference therein).

In the current literature concerning IFDEs, the impulses are assumed to take the form $\Delta x\left(t_{k}\right)=I_{k}\left(t_{k}, x\left(t_{k}^{-}\right)\right)$, which indicates that the state "jump" at the impulse times $t_{k}$ is only related to the present state variables. But in most cases, it is more applicable that the state variables on the impulses that we add are also related to the past ones. For example, in the transmission of the impulse information, input delays are often encountered (see, e.g., $[15,16])$. So, it is more meaningful if the above impulses are modified as $\Delta x\left(t_{k}\right)=$ $x\left(t_{k}\right)-x\left(t_{k}^{-}\right)=I_{k}\left(t_{k}, x\left(\left(t_{k}-d_{k}\right)^{-}\right)\right)$. Recently, there have been several attempts in the literature to study the stability and control problems of IFDEs with delayed impulse (IFDEsDI). For example, by using Lyapunov functions couples with Razumikhin techniques, some Razumikhin-type asymptotic stability and exponential stability criteria for IFDEs-DI were established in [17-19], and some Lyapunov-based sufficient conditions for the exponential stability of the equations were derived in [20].

On the other hand, stochastic perturbations are unavoidable in real equations (see [21, 22] and reference therein). In recent years, the stability analysis of impulsive stochastic functional equations which include delay equations is interesting to many investigators, and many results of stability criteria of these equations have been reported (see, e.g., [23-29]). Very recently, [30, 31] took environment noise into account and generalized delayed impulses to stochastic equations. In particular, applying the Lyapunov functions couples with Razumikhin techniques, [30] investigates both moment and almost sure exponential stability of impulsive stochastic functional differential equations with delayed impulses (ISFDEs-DI), and several Razumikhin-type criteria on the exponential stability and uniform stability in terms of two measures for the equations were established in [31]. 
But it is worth noting that the stability analysis in [30] and the effects of time delay on the impulses have been ignored. And in $[30,31]$, the authors only consider the case that the impulsive stabilization. Moreover, it is well known that the Razumikhin techniques are very effective in the study of stability problems for ordinary and functional differential equations. However, when we use the Razumikhin techniques, we need to choose an appropriate minimal class of functionals relative to which the derivative of the Lyapunov function or Lyapunov functional is estimated, which is not entirely convenient.

Motivated by the above discussion, in this paper, we will further investigate the stability of ISFDEs-DI. By using the comparison principle and an impulsive delay differential inequality, some exponential and asymptotical stability criteria are derived, which are more convenient to be applied than those Razumikhin-type conditions. Our results complement ones from some recent works and show that the ISFDE-ID will be stable if the impulses' frequency and amplitude are suitably related to the increase or decrease of the corresponding continuous stochastic flows. The rest of the paper is organized as follows. In Section 2, some relevant notations and definitions are presented. In Section 3, the comparison principle, an impulsive delay differential inequality, and several criteria on the exponential stability and asymptotical stability are established. Section 4 provides two illustrative examples to demonstrate the applications of the obtained results. Finally, conclusions are drawn in Section 5 .

\section{Preliminaries}

Throughout this paper, unless otherwise specified, we let $\left(\Omega, \mathscr{F},\left\{\mathscr{F}_{t}\right\}_{t \geqslant 0}, \mathbb{P}\right)$ be a complete probability space with a filtration $\left\{\mathscr{F}_{t}\right\}_{t \geqslant 0}$ satisfying the usual conditions; that is, it is right continuous and $\mathscr{F}_{0}$ contains all $\mathbb{P}$-null sets. Let $w(t)=\left(w_{1}(t), \ldots, w_{d}(t)\right)^{T}$ be a $d$-dimensional Brownian motion defined on the probability space. Let $\mathbb{N}$ denote the set of positive integers, $\mathbb{R}^{n}$ the $n$-dimensional real Euclidean space, and $\mathbb{R}^{n \times d}$ the space of $n \times d$ real matrices. $I$ stands for the identity matrix of appropriate dimensions. For $x \in$ $\mathbb{R}^{n},|x|$ denotes the Euclidean norm. For $A \in \mathbb{R}^{n \times d},\|A\|$ denotes spectral norm of the matrix $A$. Denote by $\lambda_{\min }(\cdot)$ the minimum eigenvalue of a matrix. If $A$ is a vector or matrix, its transpose is denoted by $A^{T}$.

Let $\tau>0$ and $\operatorname{PC}\left([-\tau, 0] ; \mathbb{R}^{n}\right)=\{\varphi:[-\tau, 0] \rightarrow$ $\mathbb{R}^{n} \mid \varphi\left(t^{+}\right)=\varphi(t)$ for all $t \in[-\tau, 0), \varphi\left(t^{-}\right)$exist and let $\varphi\left(t^{-}\right)=\varphi(t)$ for all but at most a finite number of points $t \in(-\tau, 0]\}$ be with the norm $\|\varphi\|=\sup _{-\tau \leqslant \theta \leqslant 0}|\varphi(\theta)|$, where $\varphi\left(t^{+}\right)$and $\varphi\left(t^{-}\right)$denote the right-hand and left-hand limits of function $\varphi(t)$ at $t$, respectively. Denote $\operatorname{PC}\left(\left[t_{0}-\tau, \infty\right) ; \mathbb{R}\right)=$ $\left\{\varphi|\varphi|_{\left[t_{0}-\tau, b\right]} \in \operatorname{PC}\left(\left[t_{0}-\tau, b\right] ; \mathbb{R}\right)\right.$ for all $\left.b>t_{0}-\tau\right\}$.

For $p>0$ and $t \geqslant 0$, let $\mathrm{PC}_{\mathscr{F}_{t}}^{p}\left([-\tau, 0] ; \mathbb{R}^{n}\right)$ denote the family of all $\mathscr{F}_{t}$-measurable $\operatorname{PC}\left([-\tau, 0] ; \mathbb{R}^{n}\right)$-valued random variables $\varphi$ such that $\sup _{-\tau \leqslant \theta \leqslant 0} \mathbb{E}|\varphi(\theta)|^{p}<\infty$, where $\mathbb{E}$ stands for the mathematical expectation operator with respect to the given probability measure $\mathbb{P}$. And $L_{\mathscr{F}_{t}}^{p}\left(\Omega ; \mathbb{R}^{n}\right)$ denote the family of all $\mathscr{F}_{t}$ measurable $\mathbb{R}^{n}$-valued random variables $X$, such that $\mathbb{E}|X|^{p}<\infty$. Let $\operatorname{PC}^{b}\left([-\tau, 0] ; \mathbb{R}^{n}\right)$ be the family of all bounded $\mathrm{PC}\left([-\tau, 0] ; \mathbb{R}^{n}\right)$-valued functions, and let $\mathrm{PC}_{\mathscr{F}_{t_{0}}}^{b}\left([-\tau, 0] ; \mathbb{R}^{n}\right)$ be the family of all $\mathscr{F}_{t_{0}}$ measurable $\operatorname{PC}^{b}\left([-\tau, 0] ; \mathbb{R}^{n}\right)$-valued functions.

Consider the following ISFDE-DI:

$$
\begin{aligned}
& \mathrm{d} x(t)=f\left(t, x_{t}\right) \mathrm{d} t+g\left(t, x_{t}\right) \mathrm{d} w(t), \quad t \neq t_{k}, t \geqslant t_{0}, \\
& x\left(t_{k}\right)=I_{k}\left(t_{k}, x\left(t_{k}^{-}\right), x\left(\left(t_{k}-d_{k}\right)^{-}\right)\right), \quad k \in \mathbb{N}, \\
& x_{t_{0}}(\theta)=\xi(\theta), \quad \theta \in[-\tau, 0],
\end{aligned}
$$

where the initial value $\xi \in \mathrm{PC}_{\mathscr{F}_{t_{0}}}^{b}\left([-\tau, 0] ; \mathbb{R}^{n}\right), x(t)=\left(\left(x_{1}(t)\right.\right.$, $\left.\ldots, x_{n}(t)\right)^{T}, x_{t}=x(t+\theta) \in \mathrm{PC}_{\mathscr{F}_{t}}^{p}\left([-\tau, 0] ; \mathbb{R}^{n}\right)$. Both $f: \mathbb{R}_{+} \times$ $\mathrm{PC}_{\mathscr{F}_{t}}^{p}\left([-\tau, 0] ; \mathbb{R}^{n}\right) \rightarrow \mathbb{R}^{n}$ and $g: \mathbb{R}_{+} \times \mathrm{PC}_{\mathscr{F}_{t}}^{p}\left([-\tau, 0] ; \mathbb{R}^{n}\right) \rightarrow$ $\mathbb{R}^{n \times d}$ are Borel measurable. $I_{k}: \mathbb{R}_{+} \times L_{\mathscr{F}_{t}}^{p}\left(\Omega ; \mathbb{R}^{n}\right) \times L_{\mathscr{F}_{t}}^{p}\left(\Omega ; \mathbb{R}^{n}\right)$ $\rightarrow \mathbb{R}^{n}$ represents the impulsive perturbation of $x$ at time $t_{k}$. The fixed moments of impulse times $\left\{t_{k}, k \in \mathbb{N}\right\}$ satisfy $0 \leqslant$ $t_{0}<t_{1}<\cdots<t_{k}<\cdots, t_{k} \rightarrow \infty($ as $k \rightarrow \infty) .\left\{d_{k} \geqslant 0, k \in\right.$ $\mathbb{N}\}$ are the impulse input delays satisfying $d=\sup _{k \in \mathbb{N}} d_{k}<\infty$.

As a standing hypothesis, we assume that for any $\xi \in$ $\mathrm{PC}_{\mathscr{F}_{t_{0}}}^{b}\left([-\tau, 0] ; \mathbb{R}^{n}\right)$ there exists a unique stochastic process satisfying (1) denoted by $x\left(t ; t_{0}, \xi\right)$, which is continuous on the right-hand side and limitable on the left-hand side (see [32]). Moreover, we assume that $f(t, 0) \equiv 0, g(t, 0) \equiv 0$, and $I_{k}(t, 0,0) \equiv 0$ for all $t \geqslant t_{0}, k \in \mathbb{N}$; then (1) admits a trivial solution $x(t) \equiv 0$.

We introduce the following scalar IFDE-DI as the comparison system:

$$
\begin{aligned}
& \dot{u}(t)=h\left(t, u(t), u_{t}\right), \quad t \neq t_{k}, \quad t \geqslant t_{0}, \\
& u\left(t_{k}\right)=\Psi_{1 k}\left(u\left(t_{k}^{-}\right)\right)+\Psi_{2 k}\left(u\left(t_{k}-d_{k}\right)^{-}\right), \quad k \in \mathbb{N}, \\
& u_{t_{0}}(\theta)=\zeta(\theta), \quad \theta \in[-\tau, 0],
\end{aligned}
$$

where the initial value $\zeta \in \mathrm{PC}\left([-\tau, 0] ; \mathbb{R}_{+}\right) ; u_{t} \in \mathrm{PC}([-\tau, 0]$; $\left.\mathbb{R}_{+}\right)$is defined as $u_{t}=u(t+\theta), \theta \in[-\tau, 0] . h: \mathbb{R}_{+} \times \mathbb{R}_{+} \times$ $\mathrm{PC}\left([-\tau, 0] ; \mathbb{R}_{+}\right) \rightarrow \mathbb{R}_{+}$is continuous, Lebesgue measurable, and nondecreasing with respect to the last argument; $\Psi_{1 k}$, $\Psi_{2 k}: \mathbb{R}_{+} \rightarrow \mathbb{R}_{+}$are continuous and nondecreasing. Assume that $h(t, 0,0) \equiv 0, \Psi_{1 k}(0) \equiv 0$, and $\Psi_{2 k}(0) \equiv 0$; then system (2) admits a trivial solution $u(t) \equiv 0$. We further assume that for any $\zeta \in \mathrm{PC}^{b}\left([-\tau, 0] ; \mathbb{R}_{+}\right)$, there exists a unique solution to system $(2)$ on $\left[t_{0}-\tau, \infty\right)$ denoted by $u\left(t ; t_{0}, \zeta\right)($ see $[5,6])$ which is continuous on the right-handside and limitable on the left-hand side.

For convenience, we introduce the following function classes:

$$
\begin{aligned}
& \mathscr{K}=\left\{\phi: \mathbb{R}_{+} \rightarrow \mathbb{R}_{+}\right. \text {, continuous and strictly increas- } \\
& \text { ing, } \phi(0)=0\} . \\
& \mathscr{K}_{\infty}=\{\phi \in \mathscr{K}, \phi(s) \rightarrow \infty \text { as } s \rightarrow \infty\} . \\
& C \mathscr{K}=\{\phi \in \mathscr{K}, \phi \text { is concave }\} . \\
& V \mathscr{K}_{\infty}=\left\{\phi \in \mathscr{K}_{\infty}, \phi \text { is convex }\right\} .
\end{aligned}
$$


At the end of this section, let us introduce the following definitions.

Definition 1 (see $[23,26])$. The trivial solution of (1) is said to be as follows.

(i) pth moment stable if, for any $\varepsilon>0$, there exists $\delta=$ $\delta\left(\varepsilon, t_{0}\right)>0$ such that

$$
\mathbb{E}\left|x\left(t ; t_{0}, \xi\right)\right|^{p} \leqslant \varepsilon, \quad t \geqslant t_{0},
$$

whenever $\mathbb{E}\|\xi\|^{p}<\delta$.

(ii) $p$ th moment asymptotically stable if it is $p$ th moment stable and there exists $\delta_{0}=\delta_{0}\left(t_{0}\right)$ such that

$$
\lim _{t \rightarrow \infty} \mathbb{E}\left|x\left(t ; t_{0}, \xi\right)\right|^{p}=0, \quad t \geqslant t_{0},
$$

whenever $\mathbb{E}\|\xi\|^{p}<\delta_{0}$.

(iii) $p$ th moment globally exponentially stable if there is a pair of positive constants $\lambda, C$ such that

$$
\mathbb{E}\left|x\left(t ; t_{0}, \xi\right)\right|^{p} \leqslant C \mathbb{E}\|\xi\|^{p} e^{-\lambda\left(t-t_{0}\right)}, \quad t \geqslant t_{0}
$$

for all $\xi \in \mathrm{PC}_{\mathscr{F}_{t_{0}}}^{b}\left([-\tau, 0] ; \mathbb{R}^{n}\right)$. When $p=2$, it is usually said to be globally exponentially stable in mean square.

Definition 2 (see [26]). A function $V:\left[t_{0}-\tau, \infty\right) \times \mathbb{R}^{n} \rightarrow \mathbb{R}_{+}$ belongs to class $v_{0}$ if

(i) $V$ is continuous on each of the sets $\left[t_{k-1}, t_{k}\right) \times \mathbb{R}^{n}$ and for each $x, y \in \mathbb{R}^{n}, t \in\left[t_{k-1}, t_{k}\right)$, and $k \in \mathbb{N}$, $\lim _{(t, y) \rightarrow\left(t_{k}^{-}, x\right)} V(t, y)=V\left(t_{k}^{-}, x\right)$ exists;

(ii) $V(t, x)$ is continuously once differentiable in $t$ and twice in $x$ in each of the sets $\left(t_{k-1}, t_{k}\right) \times \mathbb{R}^{n}, k \in \mathbb{N}$.

If $V \in v_{0}$, define an operator $\mathscr{L} V$ from $\left[t_{0}, \infty\right) \times$ $\mathrm{PC}\left([-\tau, 0] ; \mathbb{R}^{n}\right)$ to $\mathbb{R}$ by

$$
\begin{aligned}
\mathscr{L} V(t, \varphi)= & V_{t}(t, \varphi(0))+V_{x}(t, \varphi(0)) f(t, \varphi) \\
& +\frac{1}{2} \operatorname{trace}\left[g^{T}(t, \varphi) V_{x x}(t, \varphi(0)) g(t, \varphi)\right],
\end{aligned}
$$

where

$$
\begin{aligned}
& V_{t}(t, x)=\frac{\partial V(t, x)}{\partial t}, \\
& V_{x}(t, x)=\left(\frac{\partial V(t, x)}{\partial x_{1}}, \ldots, \frac{\partial V(t, x)}{\partial x_{n}}\right), \\
& V_{x x}(t, x)=\left(\frac{\partial^{2} V(t, x)}{\partial x_{i} \partial x_{j}}\right)_{n \times n} .
\end{aligned}
$$

\section{Main results}

In this section, we will develop an impulsive delay differential inequality and comparison principles and establish some criteria on $p$ th moment exponential stability and asymptotical stability for (1).
Lemma 3 (impulsive delay differential inequality). Assume that $c \in \mathbb{R}, \delta \in \mathbb{R}, q \in \mathbb{R}_{+}, a_{k}>0, b_{k} \geqslant 0, k \in \mathbb{N}, \bar{u}(t):=$ $\sup _{\theta \in[-\tau, 0]} u(t+\theta)$, and

(i) $\ln \left(a_{k}+b_{k} e^{c d_{k}}\right) \leqslant \delta\left(t_{k}-t_{k-1}\right)$ for each $k \in \mathbb{N}$;

(ii) $\delta+c+q \gamma<0$, where $\gamma=\sup _{k \in \mathbb{N}}\left\{e^{\delta\left(t_{k}-t_{k-1}\right)}, 1 /\right.$ $\left.e^{\delta\left(t_{k}-t_{k-1}\right)}\right\}$.

Then any solution $u \in P C\left(\left[t_{0}-\tau, \infty\right) ; \mathbb{R}_{+}\right)$of the scalar impulsive delay differential inequality problem

$$
\begin{aligned}
& D^{+} u(t) \leqslant c u(t)+q \bar{u}(t), \quad t \neq t_{k}, t \geqslant t_{0}, \\
& u\left(t_{k}\right) \leqslant a_{k} u\left(t_{k}^{-}\right)+b_{k} u\left(\left(t_{k}-d_{k}\right)^{-}\right), \quad k \in \mathbb{N}
\end{aligned}
$$

satisfies

$$
u(t) \leqslant \gamma \bar{u}\left(t_{0}\right) e^{-\lambda\left(t-t_{0}\right)}, \quad t \geqslant t_{0}-\tau,
$$

where $\lambda$ is the unique positive solution of $\lambda+\delta+c+q \gamma e^{\lambda \tau}=0$.

Proof. Set $v(t)=e^{-c\left(t-t_{0}\right)} u(t), t \in\left[t_{0}-\tau, \infty\right)$. For each $k \in \mathbb{N}$, by the second inequality of (8), we have

$$
\begin{aligned}
v\left(t_{k}\right) & =e^{-c\left(t_{k}-t_{0}\right)} u\left(t_{k}\right) \\
& \leqslant e^{-c\left(t_{k}-t_{0}\right)}\left[a_{k} u\left(t_{k}^{-}\right)+b_{k} u\left(\left(t_{k}-d_{k}\right)^{-}\right)\right] \\
& =a_{k} e^{-c\left(t_{k}-t_{0}\right)} u\left(t_{k}^{-}\right)+\beta_{k} b_{k} u\left(\left(t_{k}-d_{k}\right)^{-}\right) e^{-c\left(t_{k}-d_{k}-t_{0}\right)} \\
& =a_{k} v\left(t_{k}^{-}\right)+\beta_{k} b_{k} v\left(\left(t_{k}-d_{k}\right)^{-}\right),
\end{aligned}
$$

where $\beta_{k}=e^{c d_{k}}$.

On the other hand, for any $t \neq t_{k}, k \in \mathbb{N}$,

$$
D^{+} v(t)=e^{-c\left(t-t_{0}\right)}\left[-c u(t)+D^{+} u(t)\right] \leqslant q e^{-c\left(t-t_{0}\right)} \bar{u}(t) .
$$

For $t \in\left[t_{0}, t_{1}\right)$, integrating inequality (11) from $t_{0}$ to $t$, we obtain

$$
v(t) \leqslant v\left(t_{0}\right)+\int_{t_{0}}^{t} q e^{-c\left(s-t_{0}\right)} \bar{u}(s) \mathrm{d} s
$$

this implies that

$$
v\left(t_{1}^{-}\right) \leqslant v\left(t_{0}\right)+\int_{t_{0}}^{t_{1}} q e^{-c\left(s-t_{0}\right)} \bar{u}(s) \mathrm{d} s .
$$


For $t \in\left[t_{1}, t_{2}\right)$, by the same method, together with (10), (11), and (13), we have

$$
\begin{aligned}
v(t) \leqslant & v\left(t_{1}\right)+\int_{t_{1}}^{t} q e^{-c\left(s-t_{0}\right)} \bar{u}(s) \mathrm{d} s \\
\leqslant & a_{1} v\left(t_{1}^{-}\right)+\beta_{1} b_{1} v\left(\left(t_{1}-d_{1}\right)^{-}\right)+\int_{t_{1}}^{t} q e^{-c\left(s-t_{0}\right)} \bar{u}(s) \mathrm{d} s \\
\leqslant & a_{1}\left[v\left(t_{0}\right)+\int_{t_{0}}^{t_{1}} q e^{-c\left(s-t_{0}\right)} \bar{u}(s) \mathrm{d} s\right] \\
& +\beta_{1} b_{1}\left[v\left(t_{0}\right)+\int_{t_{0}}^{t_{1}-d_{1}} q e^{-c\left(s-t_{0}\right)} \bar{u}(s) \mathrm{d} s\right] \\
& +\int_{t_{1}}^{t} q e^{-c\left(s-t_{0}\right)} \bar{u}(s) \mathrm{d} s \\
\leqslant & \left(a_{1}+\beta_{1} b_{1}\right) v\left(t_{0}\right)+\left(a_{1}+\beta_{1} b_{1}\right) \\
& \times \int_{t_{0}}^{t_{1}} q e^{-c\left(s-t_{0}\right)} \bar{u}(s) \mathrm{d} s+\int_{t_{1}}^{t} q e^{-c\left(s-t_{0}\right)} \bar{u}(s) \mathrm{d} s .
\end{aligned}
$$

By induction, we have, for $t \in\left[t_{k-1}, t_{k}\right), k \in \mathbb{N}$,

$$
\begin{aligned}
v(t) \leqslant & v\left(t_{0}\right) \prod_{t_{0}<t_{j} \leqslant t}\left(a_{j}+\beta_{j} b_{j}\right) \\
& +\int_{t_{0}}^{t} \prod_{s<t_{j} \leqslant t}\left(a_{j}+\beta_{j} b_{j}\right) q e^{-c\left(s-t_{0}\right)} \bar{u}(s) \mathrm{d} s .
\end{aligned}
$$

Thus, for $t>t_{0}$, we get

$$
\begin{aligned}
u(t) \leqslant & u\left(t_{0}\right) e^{c\left(t-t_{0}\right)} \prod_{t_{0}<t_{j} \leqslant t}\left(a_{j}+\beta_{j} b_{j}\right) \\
& +\int_{t_{0}}^{t} \prod_{s<t_{j} \leqslant t}\left(a_{j}+\beta_{j} b_{j}\right) q e^{c(t-s)} \bar{u}(s) \mathrm{d} s .
\end{aligned}
$$

Let $t_{j_{1}}, t_{j_{2}}, \ldots, t_{j_{m}}$ be impulse points in $(s, t], t>s$. In view of condition (i), we get

$$
\begin{aligned}
\prod_{s<t_{j} \leqslant t}\left(a_{j}+\beta_{j} b_{j}\right)= & \left(a_{j_{1}}+\beta_{j_{1}} b_{j_{1}}\right) \\
& \times\left(a_{j_{2}}+\beta_{j_{2}} b_{j_{2}}\right) \cdots\left(a_{j_{m}}+\beta_{j_{m}} b_{j_{m}}\right) \\
\leqslant & e^{\delta\left(t_{j_{1}}-t_{j_{1}-1}\right)} e^{\delta\left(t_{j_{2}}-t_{j_{1}}\right)} \cdots e^{\delta\left(t_{j_{m}}-t_{j_{m-1}}\right)} \\
= & e^{\delta\left(t_{j_{m}}-t_{j_{1}-1}\right)}=e^{\delta(t-s)} e^{\delta\left(t_{j_{m}}-t\right)} e^{\delta\left(s-t_{j_{l}-1}\right)} \\
\leqslant & \gamma e^{\delta(t-s)},
\end{aligned}
$$

where $t_{j_{1}-1}$ is the first impulsive point before $t_{j_{1}}$ and satisfies $t_{j_{1}-1}<s$. Submitting this into inequality (16), then, for $t>t_{0}$,

$$
u(t) \leqslant \gamma e^{(c+\delta)\left(t-t_{0}\right)} u\left(t_{0}\right)+\int_{t_{0}}^{t} \gamma q e^{(c+\delta)(t-s)} \bar{u}(s) \mathrm{d} s .
$$

Let $\Phi(\lambda)=\lambda+c+\delta+\gamma q e^{\lambda \tau}$. Then condition (ii) implies $\Phi(0)<0$. Moreover, $\Phi(+\infty)=+\infty$ and $\Phi^{\prime}(\lambda)=1+\tau \gamma q e^{\lambda \tau}>$ 0 . Hence $\Phi(\lambda)=0$ has a unique positive solution $\lambda$. Next, we claim that

$$
u(t) \leqslant \gamma \bar{u}\left(t_{0}\right) e^{-\lambda\left(t-t_{0}\right)}, \quad t \geqslant t_{0}-\tau .
$$

Since

$$
u(t) \leqslant \bar{u}\left(t_{0}\right) \leqslant \gamma \bar{u}\left(t_{0}\right) e^{-\lambda\left(t-t_{0}\right)}, \quad t \in\left[t_{0}-\tau, t_{0}\right] .
$$

So we only need to prove (19) for $t>t_{0}$. Suppose not, then there exists a $t^{*} \in\left(t_{0},+\infty\right)$ such that

$$
\begin{gathered}
u\left(t^{*}\right)>\gamma \bar{u}\left(t_{0}\right) e^{-\lambda\left(t^{*}-t_{0}\right)}, \\
u(t) \leqslant \gamma \bar{u}\left(t_{0}\right) e^{-\lambda\left(t-t_{0}\right)}, \quad t \in\left[t_{0}-\tau, t^{*}\right) .
\end{gathered}
$$

Thus from (18), (22), and $\Phi(\lambda)=0$, we see that

$$
\begin{aligned}
u\left(t^{*}\right) \leqslant & \gamma \bar{u}\left(t_{0}\right) e^{(c+\delta)\left(t^{*}-t_{0}\right)}+\gamma \int_{t_{0}}^{t^{*}} q e^{(c+\delta)\left(t^{*}-s\right)} \bar{u}(s) \mathrm{d} s \\
\leqslant & \gamma \bar{u}\left(t_{0}\right) e^{(c+\delta)\left(t^{*}-t_{0}\right)} \\
& +\gamma \int_{t_{0}}^{t^{*}} \gamma q e^{\lambda \tau} e^{(c+\delta)\left(t^{*}-s\right)} e^{-\lambda\left(s-t_{0}\right)} \bar{u}\left(t_{0}\right) \mathrm{d} s \\
= & \gamma \bar{u}\left(t_{0}\right) e^{-\lambda\left(t^{*}-t_{0}\right)},
\end{aligned}
$$

which is a contradiction. Therefore, (19) holds. This completes the proof.

Lemma 4 (comparison principle). Assume that there exists a function $V \in v_{0}$ such that

(i) $\mathbb{E} \mathscr{L} V(t, \varphi) \leqslant h(t, \mathbb{E} V(t, \varphi(0)), \mathbb{E} V(t+\theta, \varphi))$ for any $(t, \varphi) \in\left[t_{k-1}, t_{k}\right) \times P C_{\mathscr{F}_{t}}^{p}\left([-\tau, 0] ; \mathbb{R}^{n}\right), k \in \mathbb{N} ;$

(ii) $\mathbb{E} V\left(t_{k}, I_{k}\left(t_{k}, X, Y\right)\right) \leqslant \Psi_{1 k}\left(\mathbb{E} V\left(t_{k}^{-}, X\right)\right)+\Psi_{2 k}\left(\mathbb{E} V\left(\left(t_{k}-\right.\right.\right.$ $\left.\left.\left.d_{k}\right)^{-}, Y\right)\right)$ for all $X, Y \in L_{\mathscr{F}_{t}}^{p}\left(\Omega ; \mathbb{R}^{n}\right), k \in \mathbb{N}$.

Then,

$$
\mathbb{E} V(t, x(t)) \leqslant u\left(t ; t_{0}, \zeta\right), \quad t \geqslant t_{0}
$$

provided $\mathbb{E} V\left(t_{0}+\theta, x\left(t_{0}+\theta\right)\right) \leqslant \zeta(\theta), \theta \in[-\tau, 0]$, where $x(t)=$ $x\left(t ; t_{0}, \xi\right)$ is the solution process to $(1)$.

Proof. For any $t \in\left[t_{k-1}, t_{k}\right)$ and $\alpha>0$ sufficiently small satisfying $t+\alpha<t_{k}$, by the Itô formula together with condition (i), we have

$$
\begin{aligned}
\mathbb{E} V & (t+\alpha, x(t+\alpha))-\mathbb{E} V(t, x(t)) \\
& =\int_{t}^{t+\alpha} \mathbb{E} \mathscr{L} V\left(s, x_{s}\right) \mathrm{d} s \\
& \leqslant \int_{t}^{t+\alpha} h\left(s, \mathbb{E} V(s, x(s)), \mathbb{E} V\left(s+\theta, x_{s}\right)\right) \mathrm{d} s ;
\end{aligned}
$$


this implies that

$$
\begin{aligned}
& D^{+} \mathbb{E} V(t, x(t)) \\
& \quad:=\limsup _{\alpha \rightarrow 0^{+}} \frac{\mathbb{E} V(t+\alpha, x(t+\alpha))-\mathbb{E} V(t, x(t))}{\alpha} \\
& \quad \leqslant \limsup _{\alpha \rightarrow 0^{+}} \frac{1}{\alpha} \int_{t}^{t+\alpha} h\left(s, \mathbb{E} V(s, x(s)), \mathbb{E} V\left(s+\theta, x_{s}\right)\right) \mathrm{d} s \\
& =h\left(t, \mathbb{E} V(t, x(t)), \mathbb{E} V\left(t+\theta, x_{t}\right)\right) .
\end{aligned}
$$

Write $u\left(t ; t_{0}, \zeta\right)=u(t)$ simply. Now supposing that for each $\theta \in[-\tau, 0], \mathbb{E} V\left(t_{0}+\theta, x\left(t_{0}+\theta\right)\right) \leqslant \zeta(\theta)$, we claim that

$$
\mathbb{E} V(t, x(t)) \leqslant u(t), \quad t \in\left[t_{0}-\tau, t_{1}\right) .
$$

Consider the system

$$
\begin{aligned}
& \dot{U}(t)=h\left(t, U(t), U_{t}\right)+\varepsilon, \quad t \in\left[t_{0}, t_{1}\right), \\
& U(\theta)=\zeta(\theta)+\varepsilon, \quad \theta \in\left[t_{0}-\tau, t_{0}\right],
\end{aligned}
$$

where $\varepsilon>0$ is a constant. We claim that $U(t) \geqslant \mathbb{E} V(t, x(t))$ for $t \in\left[t_{0}-\tau, t_{1}\right)$.

In fact, if this is not true, then from the continuity of $U(t)$ and $\mathbb{E} V(t, x(t))$ in $t \in\left[t_{0}, t_{1}\right)$, we know that there exist a $t^{*} \in$ $\left(t_{0}, t_{1}\right)$ and a sufficiently small constant $\alpha>0$ such that $t^{*}+$ $\alpha<t_{1}$ and

$$
\begin{aligned}
& \mathbb{E} V(t, x(t)) \leqslant U(t), \quad t \in\left[t_{0}-\tau, t^{*}\right), \\
& \mathbb{E} V\left(t^{*}, x\left(t^{*}\right)\right)=U\left(t^{*}\right), \\
& \mathbb{E} V(t, x(t))>U(t), \quad t \in\left(t^{*}, t^{*}+\alpha\right) .
\end{aligned}
$$

Thus $\dot{U}\left(t^{*}\right)=D^{+} U\left(t^{*}\right) \leqslant D^{+} \mathbb{E} V\left(t^{*}, x\left(t^{*}\right)\right)$. On the other hand, by condition (i), we obtain that

$$
\begin{aligned}
\dot{U}\left(t^{*}\right) & =h\left(t^{*}, U\left(t^{*}\right), U_{t^{*}}\right)+\varepsilon \\
& \geqslant h\left(t^{*}, V\left(t^{*}, x\left(t^{*}\right)\right), \mathbb{E} V\left(t^{*}+\theta, x_{t^{*}}\right)\right)+\varepsilon \\
& >h\left(t^{*}, V\left(t^{*}, x\left(t^{*}\right)\right), \mathbb{E} V\left(t^{*}+\theta, x_{t^{*}}\right)\right) \\
& \geqslant D^{+} \mathbb{E} V\left(t^{*}, x\left(t^{*}\right)\right) .
\end{aligned}
$$

This is a contradiction. So $U(t) \geqslant \mathbb{E} V(t, x(t))$ holds for all $t \in\left[t_{0}-\tau, t_{1}\right)$. Let $\varepsilon \rightarrow 0$; then $U(t) \rightarrow u(t)$, and hence inequality (27) holds.

Noting that $\Psi_{1 k}(\cdot)$ and $\Psi_{2 k}(\cdot)$ are nondecreasing, by (27) and condition (ii), we get

$$
\begin{aligned}
\mathbb{E} V & \left(t_{1}, x\left(t_{1}\right)\right) \\
= & \mathbb{E} V\left(t_{1}, I_{1}\left(t_{1}, x\left(t_{1}^{-}\right), x\left(t_{1}-d_{1}\right)^{-}\right)\right) \\
\leqslant & \Psi_{11}\left(\mathbb{E} V\left(t_{1}^{-}, x\left(t_{1}^{-}\right)\right)\right) \\
& +\Psi_{21}\left(\mathbb{E} V\left(\left(t_{1}-d_{1}\right)^{-}, x\left(t_{1}-d_{1}\right)^{-}\right)\right) \\
\leqslant & \Psi_{11}\left(u\left(t_{1}^{-}\right)\right)+\Psi_{21}\left(u\left(t_{1}-d_{1}\right)^{-}\right)=u\left(t_{1}\right) .
\end{aligned}
$$

Thus, it follows from (27) and (31) that

$$
\mathbb{E} V\left(t_{1}+\theta, x\left(t_{1}+\theta\right)\right) \leqslant u\left(t_{1}+\theta\right), \quad \theta \in[-\tau, 0] .
$$

Similar to the previous process, we have $\mathbb{E} V(t, x(t)) \leqslant u(t)$ when $t \in\left[t_{0}-\tau, t_{2}\right)$. By induction, it follows that $\mathbb{E} V(t, x(t)) \leqslant$ $u(t), t \in\left[t_{0}-\tau, \infty\right)$. The proof is complete.

Theorem 5. Assume that there exist functions $V \in v_{0}, \phi_{1} \in$ $V \mathscr{K}_{\infty}$, and $\phi_{2} \in C \mathscr{K}$ such that

(i) $\phi_{1}\left(|x|^{p}\right) \leqslant V(t, x) \leqslant \phi_{2}\left(|x|^{p}\right)$ for any $(t, x) \in\left[t_{0}-\right.$ $\tau, \infty) \times \mathbb{R}^{n}$

(ii) $\mathbb{E} \mathscr{L} V(t, \varphi) \leqslant h(t, \mathbb{E} V(t, \varphi(0)), \mathbb{E} V(t+\theta, \varphi))$ for any $(t, \varphi) \in\left[t_{k-1}, t_{k}\right) \times P C_{\mathscr{F}_{t}}^{p}\left([-\tau, 0] ; \mathbb{R}^{n}\right), k \in \mathbb{N} ;$

(iii) $\mathbb{E} V\left(t_{k}, I_{k}\left(t_{k}, X, Y\right)\right) \leqslant \Psi_{1 k}\left(\mathbb{E} V\left(t_{k}^{-}, X\right)\right)+\Psi_{2 k}\left(\mathbb{E} V\left(\left(t_{k}-\right.\right.\right.$ $\left.\left.\left.d_{k}\right)^{-}, Y\right)\right)$ for all $X, Y \in L_{\mathscr{F}_{t}}^{p}\left(\Omega ; \mathbb{R}^{n}\right), k \in \mathbb{N}$.

Then the stability properties of the trivial solution of IFDE-DI (2) imply the corresponding stability properties of the trivial solution of ISFDE-DI (1). Moreover, if condition (i) is replaced by

$\left(\mathrm{i}^{*}\right)$ there exist positive constants $p, c_{1}$, and $c_{2}$ such that for all $(t, x) \in\left[t_{0}-\tau, \infty\right) \times \mathbb{R}^{n}$

$$
c_{1}|x|^{p} \leqslant V(t, x) \leqslant c_{2}|x|^{p}
$$

then the global exponential stability of the trivial solution of IFDE-DI (2) implies that pth moment global exponential stability of ISFDE-DI (1).

Proof. Firstly, assume that the trivial solution of IFDE-DI (2) is stable. Let $\varepsilon>0$; then for given $\phi_{1}(\varepsilon)>0$, there exists $\delta_{1}=\delta_{1}\left(t_{0}, \varepsilon\right)>0$ such that $\delta_{1}<\phi_{1}(\varepsilon)$ and

$$
\|\zeta\|^{p}<\delta_{1} \text { implies } u\left(t ; t_{0}, \zeta\right)<\phi_{1}(\varepsilon), \quad t \geqslant t_{0} .
$$

Let $\zeta(\theta)=\mathbb{E} V\left(t_{0}+\theta, x\left(t_{0}+\theta\right)\right), \theta \in[-\tau, 0]$. From conditions (ii) and (iii) and Lemma 4 , we get that

$$
\mathbb{E} V(t, x(t)) \leqslant u\left(t ; t_{0}, \zeta\right), \quad t \geqslant t_{0} .
$$

Let $\delta \leqslant \phi_{2}^{-1}\left(\delta_{1}\right)$ and $\mathbb{E}\|\xi\|^{p}<\delta$; then by condition (i) and $\phi_{2} \epsilon$ $C \mathscr{K}$, we have $\|\zeta\|^{p} \leqslant \mathbb{E} \phi_{2}\left(\|\xi\|^{p}\right) \leqslant \phi_{2}\left(\mathbb{E}\|\xi\|^{p}\right)<\phi_{2}(\delta) \leqslant \delta_{1}$. Hence, by (34) and (35), we have

$$
\mathbb{E} V(t, x(t))<\phi_{1}(\varepsilon), \quad t \geqslant t_{0} .
$$

If $\mathbb{E}\|\xi\|^{p}<\delta$, then by conditions (i) and (36), we have

$$
\mathbb{E}|x(t)|^{p} \leqslant \phi_{1}^{-1}(\mathbb{E} V(t, x(t)))<\varepsilon, \quad t \geqslant t_{0} ;
$$

that is, the trivial solution of ISFDE-DI (1) is stable.

Next, let us suppose that the trivial solution of IFDEDI (2) is asymptotically stable. This implies that the trivial solution of ISFDE-DI (1) is stable. Let $\zeta(\theta)=\mathbb{E} V\left(t_{0}+\theta, x\left(t_{0}+\right.\right.$ $\theta)), \theta \in[-\tau, 0]$. Since $u=0$ is attractive, for any $\varepsilon>0$, there exist $\delta_{0}=\delta_{0}\left(t_{0}\right)>0$ and $T=T\left(t_{0}, \delta_{0}\right)$ such that

$$
\|\zeta\|^{p}<\delta_{0} \text {, implies } u\left(t ; t_{0}, \zeta\right)<\phi_{1}(\varepsilon), \quad t \geqslant t_{0}+T \text {. }
$$


Choose $\mathbb{E}\|\xi\|^{p}<\delta_{0}$. Note the fact that $\phi \in V \mathscr{K}$ implies $\phi^{-1} \in$ $C \mathscr{K}$. Then by (35) and (37), we get

$$
\mathbb{E}|x(t)|^{p} \leqslant \phi_{1}^{-1}(\mathbb{E} V(t, x(t)))<\varepsilon, \quad t \geqslant t_{0}+T,
$$

which implies that the trivial solution of ISFDE-DI (1) is asymptotically stable.

Thirdly, let us suppose that the trivial solution of IFDE-DI (2) is globally exponentially stable and condition $\left(i^{*}\right)$ holds. Then, there exists a couple of positive constants $\gamma$ and $K$ such that

$$
u(t) \leqslant K\|\zeta\| e^{-\gamma\left(t-t_{0}\right)}, \quad t \geqslant t_{0} .
$$

Let $\zeta(\theta)=V\left(t_{0}+\theta, x\left(t_{0}+\theta\right)\right), \theta \in[-\tau, 0]$. Then by (35) and (40), we get $\mathbb{E} V(t, x(t)) \leqslant u(t) \leqslant K \mathbb{E}\|\xi\|^{p} e^{-\gamma\left(t-t_{0}\right)}$ for all $t \geqslant t_{0}$. Thus, by condition $\left(\mathrm{i}^{*}\right)$, it yields that

$$
\mathbb{E}|x(t)|^{p} \leqslant \frac{K c_{2}}{c_{1}} \mathbb{E}\|\xi\|^{p} e^{-\gamma\left(t-t_{0}\right)}, \quad t \geqslant t_{0} .
$$

Hence, the trivial solution of ISFDE-DI (1) is pth moment globally exponentially stable. The proof is complete.

Theorem 6. Assume that there exist a function $V \in v_{0}$, positive constants $c_{1}, c_{2}, q$, and $a_{k}$, constants $c$ and $\delta$, and $b_{k} \geqslant 0$ such that

(i) $c_{1}|x|^{p} \leqslant V(t, x) \leqslant c_{2}|x|^{p}$ for any $(t, x) \in\left[t_{0}-\tau, \infty\right) \times$ $\mathbb{R}^{n}$;

(ii) $\mathbb{E} \mathscr{L} V(t, \varphi) \leqslant c \mathbb{E} V(t, \varphi(0))+q \mathbb{E} V(t+\theta, \varphi)$ for any $(t, \varphi) \in\left[t_{k-1}, t_{k}\right) \times P C_{\mathscr{F}_{t}}^{p}\left([-\tau, 0] ; \mathbb{R}^{n}\right), k \in \mathbb{N} ;$

(iii) $\mathbb{E} V\left(t_{k}, I_{k}\left(t_{k}, X, Y\right)\right) \leqslant a_{k} \mathbb{E} V\left(t_{k}^{-}, X\right)+b_{k} \mathbb{E} V\left(\left(t_{k}-\right.\right.$ $\left.\left.d_{k}\right)^{-}, Y\right)$ for all $X, Y \in L_{\mathscr{F}_{t}}^{p}\left(\Omega ; \mathbb{R}^{n}\right), k \in \mathbb{N}$;

(iv) $\ln \left(a_{k}+b_{k} e^{c d_{k}}\right) \leqslant \delta\left(t_{k}-t_{k-1}\right)$ for each $k \in \mathbb{N}$;

(v) $\delta+c+q \gamma<0$ where $\gamma=\sup _{k \in \mathbb{N}}\left\{e^{\delta\left(t_{k}-t_{k-1}\right)}, 1 / e^{\delta\left(t_{k}-t_{k-1}\right)}\right\}$.

Then the trivial solution of ISFDE-DI (1) is pth moment globally exponentially stable.

Proof. Let $u(t)=\mathbb{E} V(t, \varphi(0)), h\left(t, u(t), u_{t}\right)=c u(t)+q u_{t}$, $\Psi_{1 k}\left(u\left(t_{k}^{-}\right)\right)=a_{k} u\left(t_{k}^{-}\right)$, and $\Psi_{2 k}\left(u\left(\left(t_{k}-d_{k}\right)^{-}\right)\right)=b_{k} u\left(\left(t_{k}-\right.\right.$ $\left.\left.d_{k}\right)^{-}\right)$. We obtain the comparison system (2). It is easy to verify that all conditions of Theorem 5 are satisfied and so the global exponential stability of the trivial solution of IFDE-DI (2) implies that $p$ th moment global exponential stability of ISFDE-DI (1).

Furthermore, let $\lambda$ be the unique positive solution of $\lambda+$ $\delta+p+q \gamma e^{\lambda \tau}=0$. Using conditions (ii) and (iii), we find

$$
\begin{aligned}
& D^{+} u(t) \leqslant c u(t)+q \bar{u}(t), \quad t \neq t_{k}, t \geqslant t_{0}, \\
& u\left(t_{k}\right) \leqslant a_{k} u\left(t_{k}^{-}\right)+b_{k} u\left(\left(t_{k}-d_{k}\right)^{-}\right), \quad k \in \mathbb{N} .
\end{aligned}
$$

Thus from conditions (iv) and (v) and Lemma 3, we obtain that

$$
u(t) \leqslant \gamma \bar{u}\left(t_{0}\right) e^{-\lambda\left(t-t_{0}\right)}, \quad t \geqslant t_{0}-\tau,
$$

which implies that the trivial solution of IFDE-DI (2) is globally exponentially stable. The proof of Theorem 6 is complete.
Remark 7. An impulsive stochastic dynamical system can be viewed as a hybrid one comprised of two components: a continuous stochastic dynamic and a discrete dynamic. Theorem 6 can be used to deal will all three cases: the system with stable continuous stochastic dynamic and unstable discrete dynamic, the system with unstable continuous stochastic dynamic and stable discrete dynamic, and the system with stable continuous stochastic dynamic and stable discrete dynamic. When $c<0$, the continuous stochastic dynamic of (1) may be stable. In this case, in order to ensure the stability of the entire system, the delayed impulses' frequency $\left\{t_{k}-t_{k-1}, k \in \mathbb{N}\right\}$ and amplitude $a_{k}, b_{k}$ should be suitably related to the decrease of continuous flows; that is, conditions (iv) and (v) hold. In this sense, Theorem 6 can be used to deal with the robust stabling of continuous stochastic dynamic subject to delayed impulsive perturbations. When $c \geqslant 0$, the continuous stochastic dynamic of (1) may be unstable and the stability of the entire system is determined by the delayed impulse effects. In this case, we need to require that the delayed impulses' frequency and amplitude should be suitablly related to the decrease of of continuous flows.

Remark 8. It is noted that the exponential stability analysis in $[30,31]$ only considers the case of impulsive stabilization. In this sense, Theorem 6 has a wider adaptive range.

\section{Examples}

In this section, the effectiveness and advantages of the results derived in the preceding section will be illustrated by two examples.

Example 1. Consider the two-dimensional nonlinear impulsive stochastic delay equation in the form

$$
\begin{aligned}
\mathrm{d} x_{1}(t)= & {\left[-2 x_{2}(t) \sin \left(x_{1}(t-\tau)\right)-5 x_{1}(t)\right.} \\
& \left.+0.5 x_{2}(t-\tau)\right] \mathrm{d} t+0.2 x_{1}(t-\tau) \mathrm{d} w(t), \\
\mathrm{d} x_{2}(t)=\left[\begin{array}{l}
x_{1} \\
\quad
\end{array}\right. & \\
& \left.+0.4 x_{2}(t-\tau)\right] \mathrm{d} \sin \left(x_{1}(t-\tau)\right)-5 x_{2}(t) \\
+ & 0.4 x_{2}(t-\tau) \mathrm{d} w(t), \quad t \neq t_{k}, \\
x_{1}\left(t_{k}\right)= & x_{1}\left(t_{k}^{-}\right)+\alpha x_{1}\left(\left(t_{k}-d_{k}\right)^{-}\right), \quad k \in \mathbb{N}, \\
x_{2}\left(t_{k}\right)= & x_{2}\left(t_{k}^{-}\right)+\alpha x_{2}\left(\left(t_{k}-d_{k}\right)^{-}\right), \quad k \in \mathbb{N},
\end{aligned}
$$

where $\tau>0, d_{k} \in[0, d], \alpha \geqslant 0$. If there exists a positive constant $\varepsilon>0$ such that

$$
\begin{gathered}
\alpha<\sqrt{\frac{9 / 0.445-1-\varepsilon}{1+1 / \varepsilon}}, \\
\varrho=\inf _{k \in \mathbb{N}}\left\{t_{k}-t_{k-1}\right\}>\frac{\ln \left[1+\varepsilon+(1+1 / \varepsilon) \alpha^{2}\right]}{9-0.445\left[1+\varepsilon+(1+1 / \varepsilon) \alpha^{2}\right]},
\end{gathered}
$$


then (44) is globally exponentially stable for any bounded impulsive input delays $\left\{d_{k}\right\}$.

Denote $I_{k}\left(t_{k}, X, Y\right)=X+\alpha Y$. Choose the Lyapunov function $V(t, x)=(1 / 4) x_{1}^{2}+(1 / 2) x_{2}^{2}$; then for any $\varepsilon>0$, we have

$$
\begin{aligned}
& \mathbb{E} V\left(t_{k}, I_{k}\left(t_{k}, X, Y\right)\right) \\
&= \frac{1}{4}\left|X_{1}+\alpha Y_{1}\right|^{2}+\frac{1}{2}\left|X_{2}+\alpha Y_{2}\right|^{2} \\
&= \mathbb{E} V\left(t_{k}^{-}, X\right)+\alpha^{2} \mathbb{E} V\left(\left(t_{k}-d_{k}\right)^{-}, Y\right) \\
&+\frac{\alpha}{2} \mathbb{E}\left(X_{1} Y_{1}\right)+\alpha \mathbb{E}\left(X_{2} Y_{2}\right) \\
& \leqslant(1+\varepsilon) \mathbb{E} V\left(t_{k}^{-}, X\right)+\left(1+\frac{1}{\varepsilon}\right) \alpha^{2} \mathbb{E} V\left(\left(t_{k}-d_{k}\right)^{-}, Y\right), \\
& \mathbb{E} \mathscr{L} V(t, \varphi) \\
&=-10 \mathbb{E} V(t, \varphi(0)) \\
& \quad+\mathbb{E}\left[0.25 \varphi_{1}(0) \varphi_{2}(-\tau)+0.4 \varphi_{2}(0) \varphi_{2}(-\tau)\right. \\
&\left.\quad+0.01 \varphi_{1}^{2}(-\tau)+0.08 \varphi_{2}^{2}(-\tau)\right] \\
& \leqslant-10 \mathbb{E} V(t, \varphi(0))+\mathbb{E}\left[0.01 \varphi_{1}^{2}(-\tau)+0.08 \varphi_{2}^{2}(-\tau)\right] \\
&+\mathbb{E}\left[0.25 \varphi_{1}^{2}(0)+0.0625 \varphi_{2}^{2}(-\tau)\right. \\
&\left.+0.5 \varphi_{2}^{2}(0)+0.08 \varphi_{2}^{2}(-\tau)\right] \\
&=-9 \mathbb{E} V(t, \varphi(0))+\mathbb{E}\left[0.01 \varphi_{1}^{2}(-\tau)+0.2225 \varphi_{2}^{2}(-\tau)\right] \\
& \leqslant-9 \mathbb{E} V(t, \varphi(0))+0.445 \mathbb{E} V(t-\tau, \varphi(-\tau)),
\end{aligned}
$$

for $t \neq t_{k}$.

Take $c_{1}=1 / 4, c_{2}=1 / 2, c=-9, q=0.445, a_{k} \equiv 1+\varepsilon$, $b_{k} \equiv(1+1 / \varepsilon) \alpha^{2}, \delta=\ln \left[1+\varepsilon+(1+1 / \varepsilon) \alpha^{2}\right] / \varrho, \gamma=1+\varepsilon+$ $(1+1 / \varepsilon) \alpha^{2}$. It is easy to check that all conditions of Theorem 6 are satisfied under conditions (45), which means that (44) is globally mean square exponentially stable for any bounded impulsive input delays $\left\{d_{k}\right\}$.

Remark. It is noted that (44) without impulses is globally mean square exponentially stable and the impulses are destabilizing since $\alpha \geqslant 0$. Hence, the existing stability theorems in $[30,31]$ fail to work. This shows that our results have a wider adaptive range.

Example 2. Consider the following impulsive stochastic delayed neural network:

$$
\begin{aligned}
\mathrm{d} x(t)= & {[-x(t)+A f(x(t-\tau(t)))] \mathrm{d} t } \\
& +B x(t-\tau(t)) \mathrm{d} w(t), \quad t \neq t_{k}, \\
x\left(t_{k}\right)= & 0.3 x\left(t_{k}^{-}\right) \\
& +0.2 x\left(\left(t_{k}-d_{k}\right)^{-}\right), \quad k \in \mathbb{N},
\end{aligned}
$$

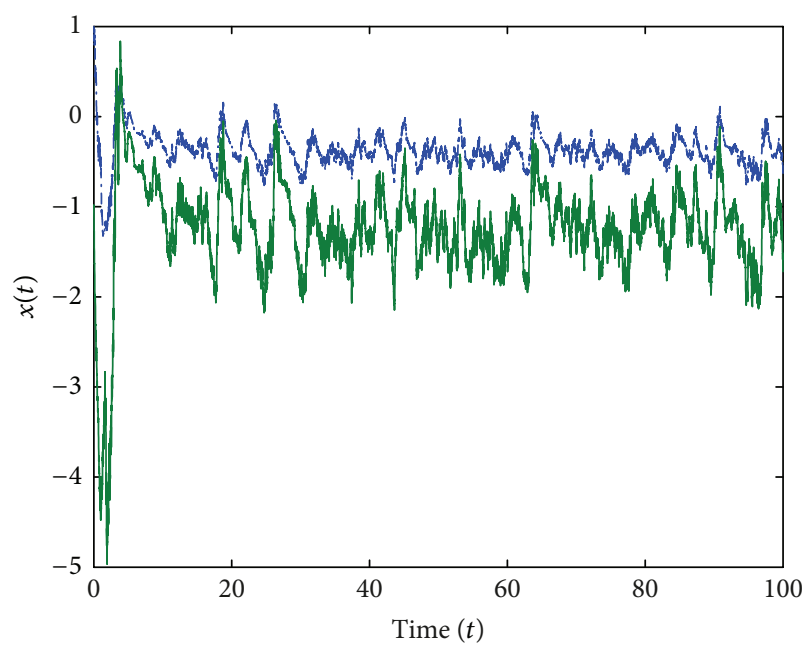

FIGURE 1: The solution of system (47) without impulses (single sample).

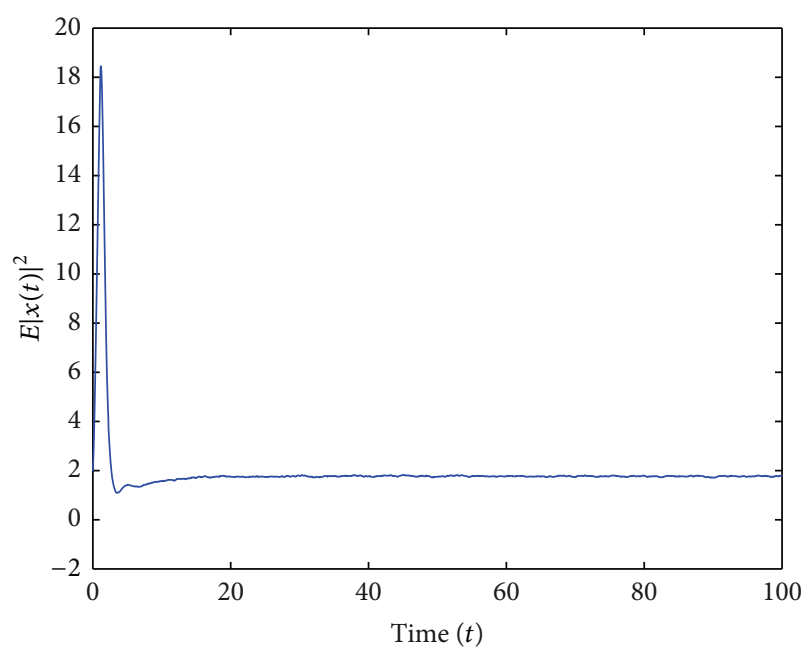

FIGURE 2: The mean square of the solution of system (47) without impulses (2000 samples).

where

$$
A=\left[\begin{array}{cc}
-1.5 & 1 \\
-3 & 2.5
\end{array}\right], \quad B=\left[\begin{array}{cc}
0.5 & 0 \\
0 & 0.4
\end{array}\right]
$$

$f(x)=\left(f_{1}\left(x_{1}\right), f_{2}\left(x_{2}\right)\right)^{T}$ with $f_{1}(s)=f_{2}(s)=(1 / 2)(|s+1|-$ $|s-1|)$.

It is noted that (47) without impulse is not stable, and its simulation with delay $\tau(t)=1$ and initial data $\xi(s)=$ $[1,-1]^{T}$ and $s \in[-1,0]$ are shown in Figures 1 and 2.

In the following, applying Theorem 5, we will show that under impulsive control law, (47) is mean square exponentially stable if $\sup _{k \in \mathbb{N}}\left\{t_{k}-t_{k-1}\right\} \leqslant 0.0681$. 


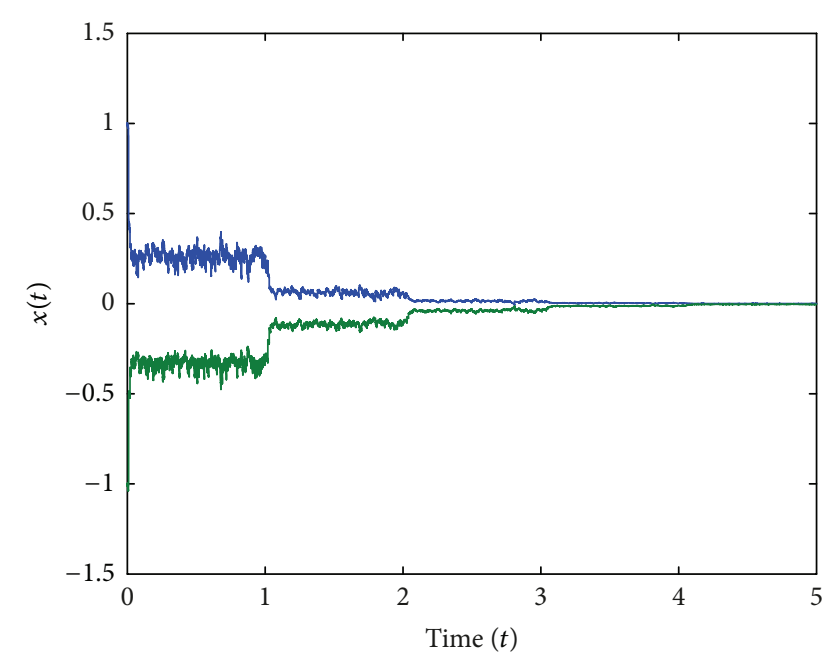

FIGURE 3: The solution of system (47) with impulses (single sample).

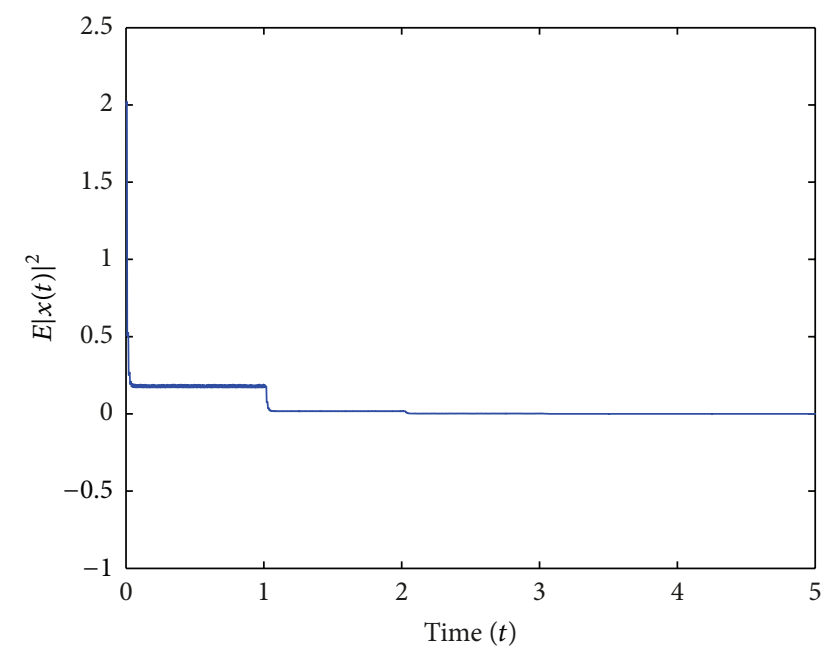

FIgURE 4: The mean square of the solution of system (47) with impulses (2000 samples).

Denote $I_{k}\left(t_{k}, X, Y\right)=0.3 X+0.2 Y$. Choose $V(t, x)=|x|^{2}$. Then condition (i) of Theorem 5 holds with $c_{1}=c_{2}=1$,

$$
\begin{aligned}
& \mathbb{E} V\left(t_{k}, I_{k}\left(t_{k}, X, Y\right)\right) \\
& =[0.3 X+0.2 Y]^{T}[0.3 X+0.2 Y] \\
& \leqslant 0.18 \mathbb{E}|X|^{2}+0.08 \mathbb{E}|Y|^{2} \\
& =0.18 \mathbb{E} V\left(t_{k}^{-}, X\right)+0.08 \mathbb{E} V\left(\left(t_{k}-d_{k}\right)^{-}, Y\right), \\
& \mathbb{E} \mathscr{L} V(t, \varphi) \\
& =\mathbb{E}\left[2 \varphi^{T}(0)(-\varphi(0)+A f(\varphi(-\tau(t))))\right] \\
& \quad+\mathbb{E}\left[\varphi^{T}(-\tau(t)) B^{T} B \varphi(-\tau(t))\right]
\end{aligned}
$$

$$
\begin{aligned}
\leqslant & \mathbb{E}\left[(-2+\|A\|)|\varphi(0)|^{2}\right. \\
& \left.+\left(\|A\|+\|B\|^{2}\right)|\varphi(-\tau(t))|^{2}\right] \\
\leqslant & 2.2976 \mathbb{E}|\varphi(0)|^{2}+4.5476 \mathbb{E}|\varphi(-\tau(t))|^{2} \\
= & 2.2976 \mathbb{E} V(t, \varphi(0)) \\
& +4.5476 \mathbb{E} V(t-\tau(t), \varphi(-\tau(t))),
\end{aligned}
$$

for $t \neq t_{k}$.

Thus, the comparison system is

$$
\begin{array}{ll}
\dot{u}(t)=2.2976 u(t)+4.5476 u(t-\tau(t)), & t \neq t_{k}, \\
& t \geqslant t_{0}, \\
u\left(t_{k}\right)=0.18 u\left(t_{k}^{-}\right)+0.08 u\left(\left(t_{k}-d_{k}\right)^{-}\right), & k \in \mathbb{N},
\end{array}
$$

which according to case (iii) of Corollary 1 in [19] is globally exponentially stable for any bounded impulsive input delays $\left\{d_{k}\right\}$ if $\sup _{k \in \mathbb{N}}\left\{t_{k}-t_{k-1}\right\}<\ln (1 / 0.26) /(2.2976+4.5476 / 0.26)=$ 0.0681 . Hence, we conclude by Theorem 6 that system (47) is mean square exponentially stable if $\sup _{k \in \mathbb{N}}\left\{t_{k}-t_{k-1}\right\} \leqslant$ 0.0681 . With the same initial value, the simulations of the impulsive stochastic delay neural network (47) under the delayed impulsive control law $x\left(t_{k}\right)=0.3 x\left(t_{k}^{-}\right)+0.2 x\left(\left(t_{k}-\right.\right.$ $\left.\left.d_{k}\right)^{-}\right), t_{k}-t_{k-1}=0.06, d_{k}=0.4$ are shown in Figures 3 and 4 .

\section{Conclusions}

This paper has investigated the exponential stability of ISFDEs-DI based on the comparison approach and an impulsive delay differential inequality. Some criteria on the pth moment global exponential stability are established. The obtained results complement some recent works. Two examples have been given to illustrate the effectiveness and the advantages of the results obtained. One of the drawbacks of the proposed method is perhaps that our results require the condition $\delta+c+q \gamma<0$ and thus cannot deal with the time delay system with $\Delta x\left(t_{k}\right)=B_{k} x\left(\left(t_{k}-d_{k}\right)^{-}\right)$. There will be future work to establish a criterion for the above system.

\section{Conflict of Interests}

The authors declare that there is no conflict of interests regarding the publication of this paper.

\section{Acknowledgments}

This work was supported by the National Natural Science Foundation of China $(11226247,11301004)$, the 211 Project of Anhui University (32030018/33010205), the Anhui Provincial Nature Science Foundation (1308085QA15/1308085MA01), the Natural Science Foundation of Jiangsu Province (BK20130239), the Research Fund for the Doctoral Program of Higher Education of China (20130094120015), the Key Foundation of Anhui Education Bureau (KJ2012A019), and 
the Research Fund for the Doctoral Program of Higher Education (20103401120002).

\section{References}

[1] V. Lakshmikantham, D. D. Bainov, and P. S. Simeonov, Theory of Impulsive Differential Equations, vol. 6 of Series in Modern Applied Mathematics, World Scientific, Singapore, 1989.

[2] A. M. Samoilenko and M. O. Perestyk, Impulsive Differential Equations, vol. 28 of Series on Advances in Mathematics for Applied Sciences, World Scientific, Singapore, 1995.

[3] K. Gopalsamy and B. G. Zhang, "On delay differential equations with impulses," Journal of Mathematical Analysis and Applications, vol. 139, no. 1, pp. 110-122, 1989.

[4] Y. V. Rogovchenko, "Impulsive evolution systems: main results and new trends," Dynamics of Continuous, Discrete and Impulsive Systems, vol. 3, no. 1, pp. 57-88, 1997.

[5] G. Ballinger and X. Z. Liu, "Existence and uniqueness results for impulsive delay differential equations," Dynamics of Continuous, Discrete and Impulsive Systems, vol. 5, no. 1-4, pp. 579-591, 1999.

[6] G. Ballinger and X. Z. Liu, "Existence, uniqueness and boundedness results for impulsive delay differential equations," Applicable Analysis, vol. 74, no. 1-2, pp. 71-93, 2000.

[7] W. M. Haddad, V. S. Chellaboina, and S. G. Nersesov, Impulsive and Hybrid Dynamical Systems: Stability, Dissipativity, and Control, Princeton Series in Applied Mathematics, Princeton University Press, Princeton, NJ, USA, 2006.

[8] A. V. Anokhin, L. Berezansky, and E. Braverman, "Exponential stability of linear delay impulsive differential equations," Journal of Mathematical Analysis and Applications, vol. 193, no. 3, pp. 923-941, 1995.

[9] I. M. Stamova and G. T. Stamov, "Lyapunov-Razumikhin method for impulsive functional differential equations and applications to the population dynamics," Journal of Computational and Applied Mathematics, vol. 130, no. 1-2, pp. 163-171, 2001.

[10] Z. G. Luo and J. H. Shen, "New Razumikhin type theorems for impulsive functional differential equations," Applied Mathematics and Computation, vol. 125, no. 2-3, pp. 375-386, 2002.

[11] Q. Wang and X. Liu, "Impulsive stabilization of delay differential systems via the Lyapunov-Razumikhin method," Applied Mathematics Letters, vol. 20, no. 8, pp. 839-845, 2007.

[12] W. H. Chen and W. X. Zheng, "Robust stability and $H_{\infty}$-control of uncertain impulsive systems with time-delay," Automatica, vol. 45, no. 1, pp. 109-117, 2009.

[13] X. L. Fu and X. D. Li, "Razumikhin-type theorems on exponential stability of impulsive infinite delay differential systems," Journal of Computational and Applied Mathematics, vol. 224, no. 1, pp. 1-10, 2009.

[14] X. D. Li, "New results on global exponential stabilization of impulsive functional differential equations with infinite delays or finite delays," Nonlinear Analysis-Real World Applications, vol. 11, no. 5, pp. 4194-4201, 2010.

[15] F. L. Lian, J. Moyne, and D. Tilbury, "Modelling and optimal controller design of networked control systems with multiple delays," International Journal of Control, vol. 76, no. 6, pp. 591606, 2003.

[16] A. Khadra, X. Z. Liu, and X. M. Shen, "Analyzing the robustness of impulsive synchronization coupled by linear delayed impulses," IEEE Transactions on Automatic Control, vol. 54, no. 4, pp. 923-928, 2009.

[17] Y. Zhang and J. T. Sun, "Stability of impulsive functional differential equations," Nonlinear Analysis-Theory, Methods \& Applications, vol. 68, no. 12, pp. 3665-3678, 2008.

[18] P. Cheng, Z. Wu, and L. L. Wang, "New results on global exponential stability of impulsive functional differential systems with delayed impulses," Abstract and Applied Analysis, vol. 2012, Article ID 376464, 13 pages, 2012.

[19] D. W. Lin, X. D. Li, and D. O'Regan, "Stability analysis of generalized impulsive functional differential equations," Mathematical and Computer Modelling, vol. 55, no. 5-6, pp. 1682-1690, 2012.

[20] W. H. Chen and W. X. Zheng, "Exponential stability of nonlinear time-delay systems with delayed impulse effects," Automatica, vol. 47, no. 5, pp. 1075-1083, 2011.

[21] X. R. Mao, Stochastic Differential Equations and Applications, Horwood, Chichester, UK, 1997.

[22] A. M. Samoilenko and O. M. Stanzhytskiy, Qualitative and Asymptotic Analisis of Differential Equations with Random Peryurbations, vol. 78 of World Scientific Series on Nonlinear Science, Series A, 2011.

[23] S. G. Peng and B. G. Jia, "Some criteria on pth moment stability of impulsive stochastic functional differential equations," Statistics \& Probability Letters, vol. 80, no. 13-14, pp. 1085-1092, 2010.

[24] P. Cheng and F. Q. Deng, "Global exponential stability of impulsive stochastic functional differential systems," Statistics \& Probability Letters, vol. 80, no. 23-24, pp. 1854-1862, 2010.

[25] C. X. Li, J. T. Sun, and R. Y. Sun, "Stability analysis of a class of stochastic differential delay equations with nonlinear impulsive effects," Journal of the Franklin Institute, vol. 347, no. 7, pp. 11861198, 2010.

[26] S. G. Peng and Y. Zhang, "Razumikhin-type theorems on pth moment exponential stability of impulsivestochastic delay differential equations," IEEE Transactions on Automatic Control, vol. 55, no. 8, pp. 1085-1092, 2010.

[27] L. J. Pan and J. D. Cao, "Exponential stability of impulsive stochastic functional differential equations," Journal of Mathematical Analysis and Applications, vol. 382, no. 2, pp. 672-685, 2011.

[28] F. Q. Yao and F. Q. Deng, "Exponential stability in terms of two measures of impulsive stochastic functional differential systems via comparison principle," Statistics \& Probability Letters, vol. 82, no. 6, pp. 1151-1159, 2012.

[29] F. Q. Yao and F. Q. Deng, "Stability of impulsive stochastic functional differential systems in terms of two measures via comparison approach," Science China Information Sciences, vol. 55, no. 6, pp. 1313-1322, 2012.

[30] J. Liu, X. Z. Liu, and W. C. Xie, "Impulsive stabilization of stochastic functional differential equations," Applied Mathematics Letters, vol. 24, no. 3, pp. 264-269, 2011.

[31] F. Q. Yao, F. Q. Deng, and P. Cheng, "Exponential stability of impulsive stochastic functional differential systems with delayed impulses," Abstract and Applied Analysis, vol. 2013, Article ID 548712, 8 pages, 2013.

[32] M. S. Alwan, X. Z. Liu, and W. C. Xie, "Existence, continuation, and uniqueness problems of stochastic impulsive systems with time delay," Journal of the Franklin Institute, vol. 347, no. 7, pp. 1317-1333, 2010. 


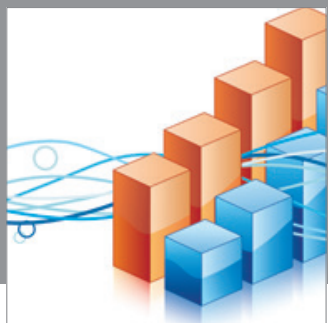

Advances in

Operations Research

mansans

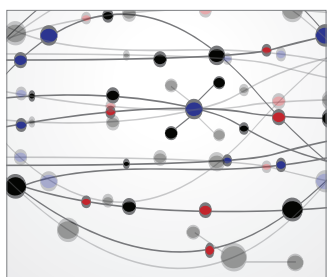

The Scientific World Journal
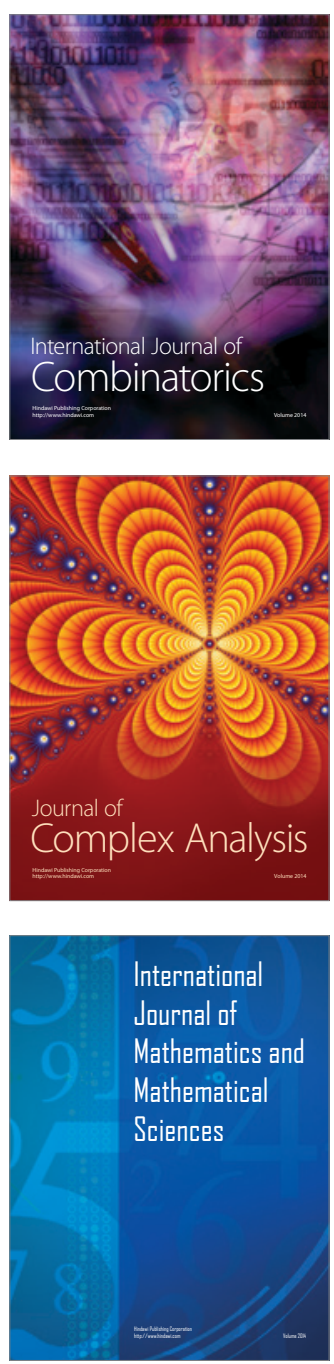
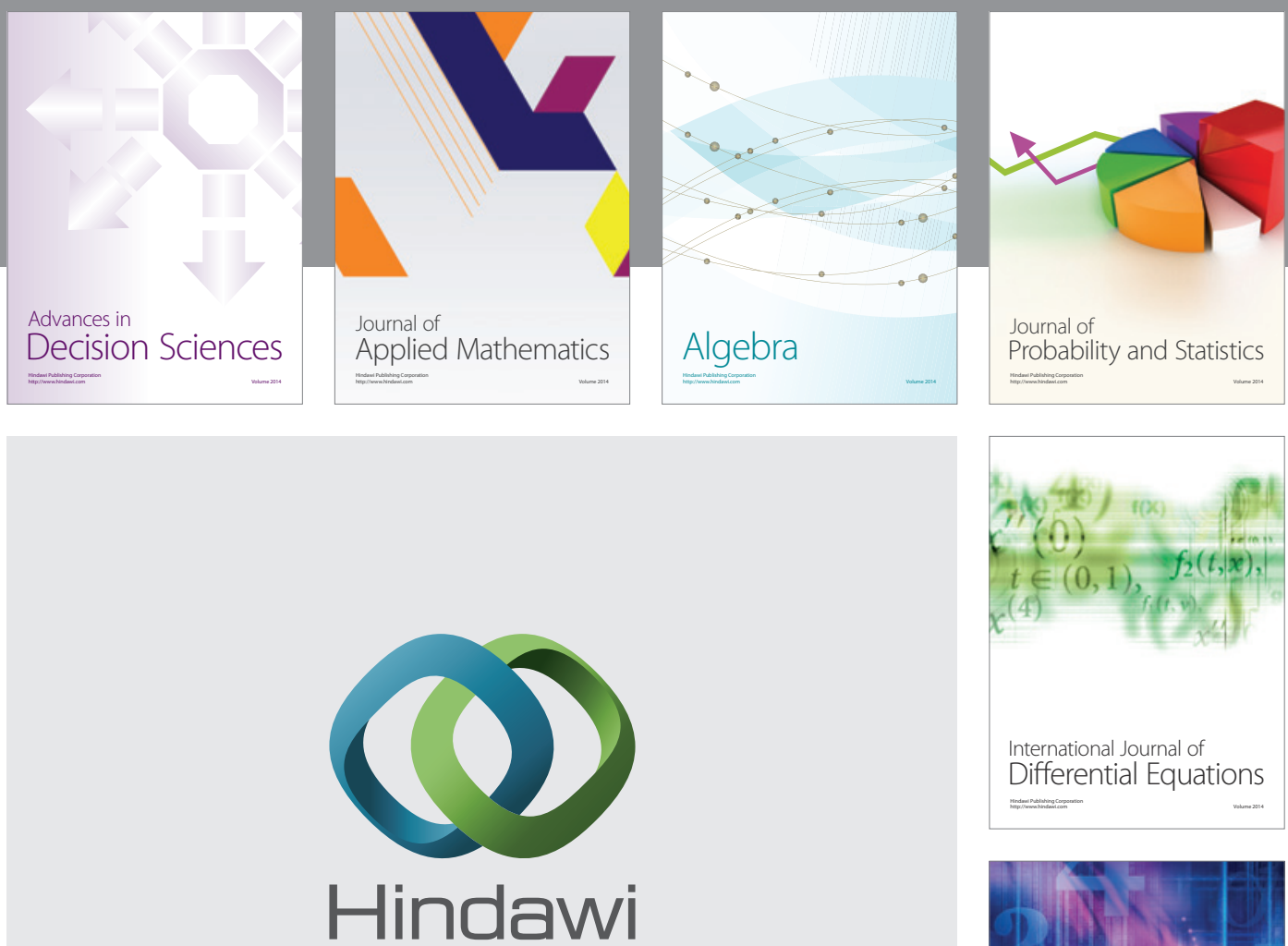

Submit your manuscripts at http://www.hindawi.com
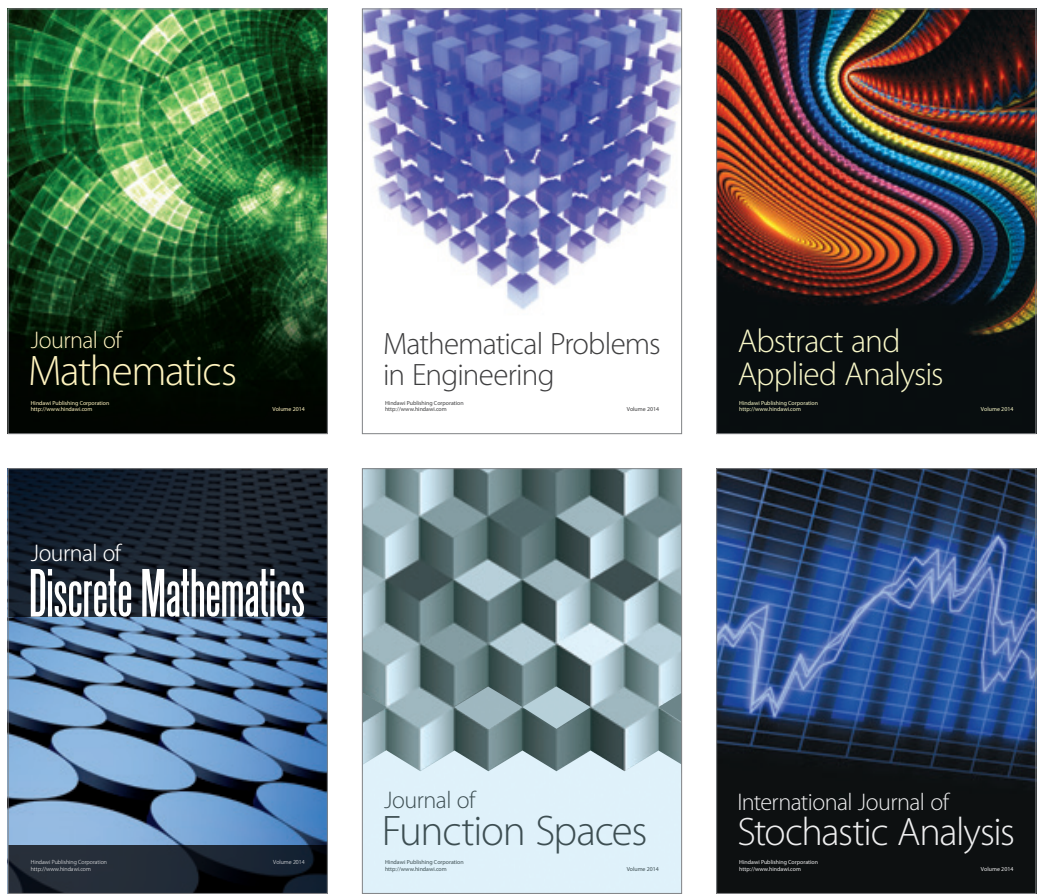

Journal of

Function Spaces

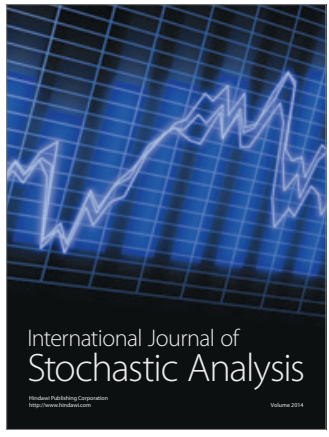

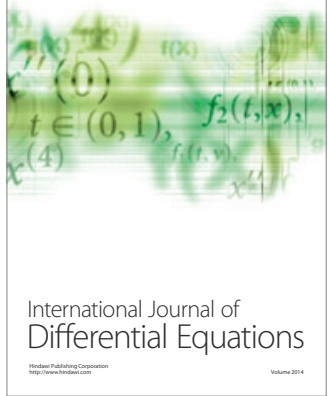
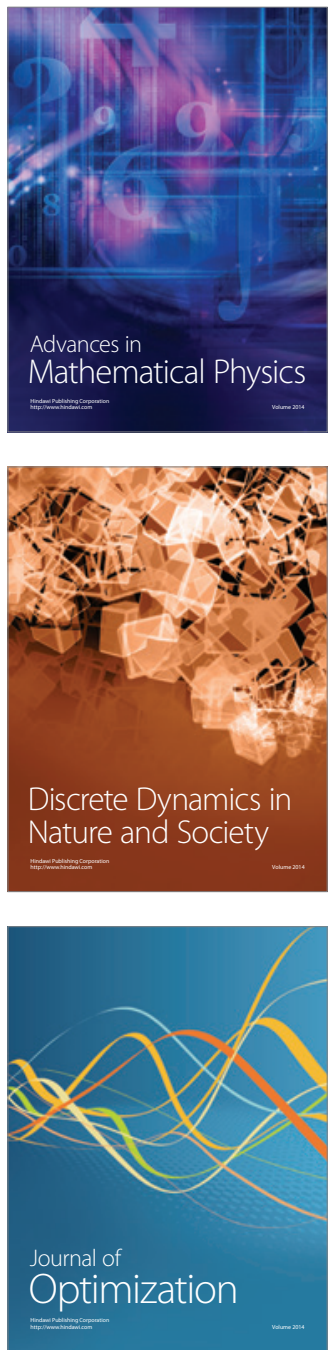